\section{(6) OPEN ACCESS}

\title{
International Committee on Mental Health in Cystic Fibrosis: Cystic Fibrosis Foundation and European Cystic Fibrosis Society consensus statements for screening and treating depression and anxiety
}

\author{
Alexandra L Quittner, ${ }_{1}^{1}$ Janice Abbott, ${ }^{2}$ Anna M Georgiopoulos, ${ }^{3}$ Lutz Goldbeck, ${ }^{4}$ \\ Beth Smith, ${ }^{5}$ Sarah E Hempstead, ${ }^{6}$ Bruce Marshall, ${ }_{1}^{7}$ Kathryn A Sabadosa, ${ }^{6}$ \\ Stuart Elborn, ${ }^{8}$ the International Committee on Mental Health
}

- Additional material is published online only. To view, please visit the journal online (http://dx.doi.org/10.1136/ thoraxjnl-2015-207488)

For numbered affiliations see end of article.

\section{Correspondence to Dr Alexandra L Quittner, Department of Psychology, University of Miami, 5665 Ponce de Leon Blvd., Coral Gables, FL 33146, USA; aquittner@miami.edu}

Received 24 June 2015 Revised 26 August 2015 Accepted 5 September 2015 Published Online First 9 October 2015

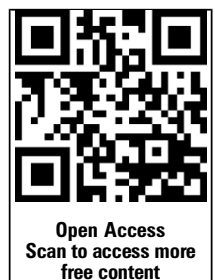

\section{SLinked}

- http://dx.doi.org/10.1136/ thoraxjnl-2015-208076

To cite: Quittner $A L$ Abbott J,

Georgiopoulos AM, et al. Thorax 2016;71:26-34.

\section{ABSTRACT}

Studies measuring psychological distress in individuals with cystic fibrosis (CF) have found high rates of both depression and anxiety. Psychological symptoms in both individuals with CF and parent caregivers have been associated with decreased lung function, lower body mass index, worse adherence, worse health-related quality of life, more frequent hospitalisations and increased healthcare costs. To identify and treat depression and anxiety in CF, the CF Foundation and the European CF Society invited a panel of experts, including physicians, psychologists, psychiatrists, nurses, social workers, a pharmacist, parents and an individual with $C F$, to develop consensus recommendations for clinical care. Over 18 months, this 22-member committee was divided into four workgroups: Screening; Psychological Interventions; Pharmacological Treatments and Implementation and Future Research, and used the Population, Intervention, Comparison, Outcome methodology to develop questions for literature search and review. Searches were conducted in PubMed, PsychINFO, ScienceDirect, Google Scholar, Psychiatry online and ABDATA by a methodologist at Dartmouth. The committee reviewed 344 articles, drafted statements and set an $80 \%$ acceptance for each recommendation statement as a consensus threshold prior to an anonymous voting process. Fifteen guideline recommendation statements for screening and treatment of depression and anxiety in individuals with CF and parent caregivers were finalised by vote. As these recommendations are implemented in CF centres internationally, the process of dissemination, implementation and resource provision should be closely monitored to assess barriers and concerns, validity and use.

\section{INTRODUCTION}

Original studies, meta-analyses and systematic reviews have shown that adults and children with chronic conditions, as well as parent caregivers, are at higher risk for depression and anxiety in comparison with community samples ${ }^{12}$ (see online supplementary appendix A). Cystic fibrosis (CF), a common genetic, life-shortening chronic illness, leading to frequent infections and progressive

\section{Key messages}

What is the key question?

- Given the high prevalence of depression and anxiety among individuals with cystic fibrosis (CF) and parent caregivers, the International Committee on Mental Health in CF (ICMH) tackled the question of how we can change clinical practice to improve mental health outcomes.

\section{What is the bottom line?}

- The ICMH is recommending that when annual screening shows elevated levels of depression and anxiety, clinical diagnostic procedures should be implemented, followed by evidence-based psychological and/or pharmacological interventions, if needed.

\section{Why read on?}

- International implementation of the guidelines, which were developed over nearly 3 years by a number of international experts, will address the needs of individuals with CF and parent caregivers, and will likely improve their health outcomes and quality of life.

failure of most organ systems (eg, lungs, pancreas) was the focus of our study. ${ }^{3}$ Despite recent advances in diagnosis and treatment, management of $\mathrm{CF}$ requires a complex, time-consuming daily regimen taking $2-4 \mathrm{~h} /$ day. ${ }^{4}$ Thus, CF continues to be one of the most difficult chronic conditions to manage.

There is strong consensus that depression is defined as "a mood disorder that affects the way a person feels, thinks or behaves, which impairs social or occupational functioning" (ref., 5 p.775). Central to this is depressed mood or loss of interest in most activities. ${ }^{6}$ Risk for suicide is a core component of depression, is a major cause of death among adolescents and adults in the general population and in recent Cystic Fibrosis Foundation (CFF) Registry data, $1.6 \%$ of deaths were 
categorised as explicit suicide. ${ }^{7}$ Anxiety is a "state of intense apprehension, uncertainty, and fear resulting from the anticipation of a threatening event or situation to the degree that normal physical and psychological functioning is disrupted." 8 Procedural anxiety, which is particularly important for individuals with $\mathrm{CF}$, has been defined as an acute and excessive fear of a medical or surgical procedure that results in acute stress or avoidance. Patients may experience anxiety in anticipation of or during procedures. Avoidance due to procedural anxiety can have negative health consequences. ${ }^{9}$

Studies measuring psychological distress in individuals with CF have found high rates of both depression and anxiety. The prevalence of depression ranges from $8 \%$ to $29 \%$ among children and adolescents, and 13-33\% among adults; $;^{10}{ }^{11}$ anxiety in adults has ranged from $30 \%$ to $33 \% .^{7}$ Caregivers have also reported elevations in depression scores ranging from $20 \%$ to $35 \% .^{12} \mathrm{~A}$ recent study in nine countries screened 6088 patients with CF ages 12 years through adulthood and 4102 parents. ${ }^{13}$ Elevated symptoms of depression were found in 130 adolescents (10\%), 913 adults (19\%), 1165 mothers (37\%), and 305 fathers (31\%). Anxiety was reported by 281 adolescents (22\%), 1503 adults (32\%), 1496 mothers (48\%), and 343 fathers (36\%). Elevations were 2-3 times those reported in community samples. High rates of comorbidity were found between depression and anxiety symptoms across patient and parent samples. Further, among 1122 parent-teen dyads, adolescents were more than twice as likely to report elevated depression or anxiety if either parent was elevated.

Importantly, psychological symptoms in both patients and parents have been associated with decreased lung function, ${ }^{14}$ lower body mass index, ${ }^{15}$ worse adherence, ${ }^{10}{ }^{16}$ worse health-related quality of life, ${ }^{17}$ more frequent hospitalisations and increased healthcare costs ${ }^{15}$ (see online supplementary appendix B). Given these high rates of depression and anxiety and their effects on quality of life and key health outcomes, the CFF and the European Cystic Fibrosis Society (ECFS) supported the formation of an International Committee on Mental Health in CF (ICMH).

\section{METHODS}

The CFF and ECFS formed a 22-member multidisciplinary committee including professionals, parents of individuals with CF and an adult with CF by invitation. They met in May 2013 and elected to focus on the assessment and treatment of depression and anxiety. Four workgroups were created: Screening; Psychological Interventions; Pharmacological Treatments and Implementation and Future Research. Each workgroup developed topic-specific questions using the Population, Intervention, Comparison, Outcome (PICO) format. ${ }^{18}$ These questions were reviewed and approved by the entire committee before literature searches were conducted.

The PICO questions were used to guide literature searches in PubMed, PsychINFO, ScienceDirect, Google Scholar, Psychiatry online and ABDATA conducted by a methodologist at the Dartmouth Institute of Health Policy and Clinical Practice at the Geisel School of Medicine at Dartmouth. Searches were limited to the English language and the period of 1960-2015. Standard textbooks were also consulted. Additional topic-relevant guidelines and reviews targeting general, chronically ill and CF-specific populations were identified through searches of the websites of organisations, including American Thoracic Society, Cochrane Collaboration, American Academy of Pediatrics, Agency for Healthcare Research and Quality, National Health and Medical Research Council, Canadian Psychiatric Association, National Institute for Health Care Excellence,
American Academy of Child and Adolescent Psychiatry, American Psychiatric Association, British Association for Psychopharmacology, World Federation of Societies of Biological Psychiatry and US Department of Veterans Affairs.

In April 2014, the committee reconvened to finalise the recommendation statements. A threshold consensus vote of $80 \%$ acceptance for each statement was set prior to the meeting. At the meeting, each workgroup presented draft recommendation statements and rationale to the full committee. After review and discussion of each statement, committee members voted anonymously. Review and voting continued until the $80 \%$ or higher acceptance rate was reached for each statement.

A preliminary version of the manuscript was distributed for public comment to the CF clinical, parent and patient communities in Europe and the USA. Feedback and comments were collected via a web-based survey. The committee reviewed and responded to this feedback.

\section{RESULTS}

A total of 21756 references were retrieved in the creation of these recommendation statements. Of those, 980 abstracts and books were reviewed, and 344 articles were chosen for more in-depth review. Articles were excluded if they focused on children under the age of 7 because there is less evidence supporting screening and treatment of depression and anxiety in this age group. However, attention to the mental health of families from the time of diagnosis is recommended by other guidelines ${ }^{19}$ and in this review, we included articles on parents of children with CF from birth to adulthood. After review, key articles were selected to address the PICO questions under consideration by the committee. Articles not referenced in this document appear in the online supplementary appendices. Fifteen guideline recommendation statements for screening and treatment of depression and anxiety in individuals with $\mathrm{CF}$ and parent caregivers were finalised by vote (table 1 ).

\section{PREVENTION}

In the course of routine care, all individuals with CF and caregivers should be offered education and preventative, supportive interventions to promote effective coping skills and disease management. Care teams should provide support in a sensitive and empathic manner, paying attention to individual and family functioning, encouraging habits that promote good physical and mental health-including CF centres that are already using preventive interventions every day from the time of first diagnosis through the end of life, in the form of providing education about $\mathrm{CF}$ in a sensitive and empathetic manner, paying attention to individual and family functioning and coping and encouraging habits that promote good physical and mental healthincluding exercise, good nutrition, sleep hygiene and finding ways to balance the demands of CF with education, work and pleasurable activities that make life satisfying and meaningful. ${ }^{20}$ Specific preventive strategies may be developed to reduce the risk of anxiety and depression in CF. For example, training in specific problem-solving and cognitive behavioural skills can decrease anxiety and improve resilience ${ }^{9}{ }^{21}$ See Recommendation 1 in table 1.

In addition, there are a number of painful medical procedures experienced by patients with $\mathrm{CF}$, including blood draws and intravenous or peripherally inserted central catheter insertions, and behavioural approaches can reduce distress related to these procedures. ${ }^{9}$ For individuals with $\mathrm{CF}$ undergoing medical procedures, the ICMH recommends that behavioural approaches be used to reduce the risk of distress. See Recommendation 2 in table 1. 
1. For all individuals with CF and caregivers, the CFF/ECFS International Committee on Mental Health in CF (ICMH) recommends that ongoing education and preventative, supportive interventions, such as training in stress management and the development of coping skills, aligned with appropriate developmental stage and disease events be offered.

2. For all individuals with CF undergoing medical procedures, the ICMH recommends that behavioural approaches be used to reduce the risk of distress.

Screening

3. The ICMH recommends that children with CF ages 7-11 be clinically evaluated for depression and anxiety when caregiver depression or anxiety scores are elevated, or when significant symptoms of depression or anxiety in the child are reported or observed by patients, caregivers or members of the CF multidisciplinary team

4. The ICMH recommends annual screening for depression and anxiety with the PHQ-9 and GAD-7 for adolescents and adults with CF (ages $12-$ adulthood).

5. The ICMH recommends offering annual screening for depression and anxiety to at least one primary caregiver of children and adolescents with CF (ages 0-17) using one of the following approaches listed below, depending on staffing and resources:

- Screening with the PHQ-9 and GAD-7

- Screening with the PHQ-8 and GAD-7

- Screening with the PHQ-2 and GAD-2

Clinical Assessment

6. The ICMH recommends that any treatment for depression and anxiety in individuals with CF and caregivers be based on clinical diagnosis.

- A healthcare provider with appropriate training and expertise should evaluate the clinical significance of elevated screening scores and presenting symptoms to perform a differential diagnosis before initiating treatment.

7. For caregivers of individuals with CF who have clinically significant symptoms of depression/anxiety, the ICMH recommends referral for treatment to primary care or mental health services after initial assessment with the CF team.

Intervention

8. For all individuals with CF and symptoms of depression/anxiety, the ICMH recommends a flexible, stepped care model of clinical intervention developed and implemented in close collaboration with patients and caregivers, the multidisciplinary CF team and other treatment providers or consultants, such as primary care or mental health specialists.

- CF teams must identify who will be responsible to initiate and coordinate care and monitor treatment effects.

9. The ICMH recommends that in children with CF ages 7-11, who have clinically significant depression or anxiety, evidence-based psychological interventions are recommended as the first-line treatment.

10. For individuals with CF ages 12-adulthood and mild depression or anxiety symptoms, the ICMH recommends education about depression/anxiety, preventative or supportive interventions and rescreening at the next clinic visit.

11. For individuals with CF ages 12-adulthood and moderate depression or anxiety, the ICMH recommends offering or providing a referral for evidence-based psychological interventions, including CBT or IPT.

- When psychological intervention is unavailable, declined or not fully effective, antidepressant treatment should be considered.

12. For individuals with CF ages 12-adulthood and severe depression, the ICMH recommends use of combined evidence-based psychological interventions and antidepressant pharmacotherapy.

13. For individuals with CF ages 12-adulthood and severe anxiety, the ICMH recommends offering exposure-based CBT.

- When exposure-based CBT is unavailable, declined or not fully effective, antidepressant medications can be considered.

14. The ICMH recommends that the SSRIs citalopram, escitalopram, sertraline and fluoxetine are appropriate first-line antidepressants for most individuals with $\mathrm{CF}$, ages 12-adulthood, requiring pharmacotherapy.

- In selecting an antidepressant and adjusting its dosage, close monitoring of therapeutic effects, adverse effects, drug-drug interactions and medical comorbidities is recommended.

15. The ICMH recommends that lorazepam be considered for short-term use in individuals with CF with moderate-to-severe anxiety symptoms, associated with medical procedures, who have not responded to behavioural approaches.

CBT, cognitive behavioural therapy; CF, cystic fibrosis; CFF, Cystic Fibrosis Foundation; ECFS, European Cystic Fibrosis Society; GAD, Generalised Anxiety Disorder Questionnaire; IPT, interpersonal therapy; PHQ, Patient Health Questionnaire; SSRIs, selective serotonin reuptake inhibitors.

\section{SCREENING}

Care pathways and provision of high-quality care for depression/anxiety should be in place prior to implementation of a screening programme. ${ }^{22}$ Given that models of healthcare delivery and availability of resources differ worldwide, it is difficult to specify the qualifications and training of the team member who will assess and treat mental health issues. ${ }^{22}$ In Europe, CF teams can consult the ECFS Standards of Care documents, ${ }^{20}$ but in the USA this document does not exist and the designated professional may be a social worker, nurse practitioner, psychologist or psychiatrist. Before initiating annual screening, CF Teams should (1) identify a clinician with specialised expertise and training in mental health (e.g., licensed social worker, psychologist, psychiatrist), (2) develop educational materials on the importance of assessing and treating depression and anxiety and (3) develop a list of referral sources within the hospital and surrounding community. A plan to address suicidal ideation, which can be associated with depression, should also be established (eg, clinical assessment or interview, visit to emergency department). See online supplementary appendix C for a Manual of Procedures and Toolkit for Implementation.

Evidence from The International Depression Epidemiological Study (TIDES) indicated that when a parent reported elevated depressive or anxious symptoms, the adolescent with CF was more than twice as likely to also experience depression and anxiety. ${ }^{13}$ There is limited guidance on routine screening of younger children below age 12 . Thus, for children with $\mathrm{CF}$, ages $7-11$, whose parents score in the elevated range or who themselves have clinical concerns, referrals for screening or clinical assessment for these younger children should be made to mental health experts in the CF team, in the hospital or in the community. See Recommendation 3 in table 1.

The US Screening Guidelines recommend universal screening for depression in adolescents and adults and the UK 
recommends screening for high-risk populations. ${ }^{6} 5^{5} \quad 23-26$ In addition, chronic disease practice guidelines ${ }^{9}$ recommend mental health screening because of their link to worse health outcomes. These practice guidelines, in combination with recent screening evidence in $\mathrm{CF}^{13}$ support our recommendation for annual screening of depression and anxiety in patients with $\mathrm{CF}$ ages 12 years and older and parent caregivers of children with CF aged from birth to 17 years of age.

Internationally, more than 48 different screening tools were in use at CF centres. ${ }^{22}$ To standardise the screening process using reliable, valid measures that yield clinically relevant scores, the committee recommends the use of the Patient Health Questionnaire 9 (PHQ-9), which includes an item to assess suicide risk, and Generalised Anxiety Disorder 7-item (GAD-7) Scale for annual screening of adolescents (ages 12 years and older) and adults with CF and offered annually to at least one primary caregiver of children with CF (ages 0-17 years). The PHQ-9 and GAD-7 are free, brief, reliable and valid, with optimal cut-off scores for detecting psychological symptoms, map onto current diagnostic criteria Diagnostic and Statistical Manual of Mental Disorders, 5th Edition (DSM-5)) ${ }^{8}$ and are available in all major languages.

Guidelines from US and UK societies advocate for the use of PHQ-9 when appropriate diagnostic, treatment and follow-up services are available. ${ }^{6} 242527$ The PHQ-9 is also recommended for depression severity assessment. ${ }^{8}$ When screening anxiety, UK societies endorse the use of the GAD-7. ${ }^{28}$ Figure 1 outlines the meta-strategy for screening and treating depression and anxiety.

A brief explanation and discussion of the rationale for screening mental health issues is recommended for all patients and parent caregivers, followed by administration of the PHQ-9 and GAD-7 (http://www.phqscreeners.com/). For caregivers, centres that do not have the resources or expertise to assess suicidality may choose to omit the question on the PHQ-9 that assesses self-harm and administer the PHQ-8. An alternative approach is to use two items from the PHQ-9 on low mood and anhedonia (PHQ-2, http://www.cqaimh.org/pdf/tool_phq2.pdf) and two items from the GAD-7 on feeling anxious/nervous and not being able to stop or control worrying (GAD-2, http:// depression.acponline.org/content/all/tools/dcg_o11.pdf). See Recommendations 4 and 5 in table 1.

\section{CLINICAL ASSESSMENT}

We recommend that a mental health specialist (eg, a licensed social worker, psychologist, psychiatrist) perform the screening. However, there may be other providers (eg, nurse, physician), with additional training or consultation, who can conduct the screening. Individuals with CF who screen positive for depression/anxiety must have a clinical assessment prior to initiation of or referral for treatment to identify the presence, duration and severity of these symptoms, as well as prior history and risk factors. Severity of CF, prior history of depression or anxiety, previous treatment and response to treatment(s), family history of psychiatric illnesses, comorbid psychiatric diagnoses and the presence of other chronic illnesses should be assessed. Treatment decisions should be based on clinical diagnosis and not solely on the screening results. CF healthcare professionals should decide when referral to a trained mental health professional is required.

All caregivers who screen positive and have clinically significant symptoms of depression/anxiety should be advised to follow up with their primary care provider or mental health services (outside the CF centre), with provision of referrals as needed. Although some CF centres may have the expertise and resources to treat parental depression and anxiety within their centre, this will not be the case in a majority of countries. Thus, we are recommending provision of psychological services for parents outside the CF team.

For patients or caregivers who screen positive for suicide risk (Question 9 on the PHQ-9), the designated mental health expert in the CF team should follow up immediately to determine the severity. This should include a clinical interview or further assessment. There are formal tools, such as the Columbia Suicide Severity Rating Scale (C-SSRS; http:/www. ccrs.columbia.edu/ecssrs.hmtl), which can also be used to evaluate this risk. This measure is free, well validated and available in over 100 languages. This tool was designed for use by 'lay professionals' (eg, teacher, law enforcement) and has an on-line training course and certification available. It is appropriate for children, adolescents and adults. ${ }^{29}$ See Recommendations 6 and 7 in table 1.

\section{INTERVENTION}

A variety of international authorities and professional associations have issued evidence-based guidelines for the treatment of depression and anxiety in the general population and in those with chronic illness (see online supplementary appendix D tables 1 and 2). Given that minimal research has specifically examined the treatment of depression and anxiety in $\mathrm{CF}^{30}$ the ICMH used developmentally appropriate, existing guidelines to frame CF-specific recommendations. Although treatment plans should consider potential adverse effects and overall burden of care, the risks of not treating depression or anxiety are often heightened in individuals with CF leading to worse adherence to $\mathrm{CF}$ treatments and increased healthcare utilisation and costs. $^{10} 1517$

Treatment plans for depression/anxiety must be developed and implemented in close collaboration with patients and caregivers, the multidisciplinary $\mathrm{CF}$ healthcare professionals and other providers, such as primary care or mental health specialists. Figures 2 and 3 illustrate a flexible stepped care model for prevention, screening and intervention for individuals with $\mathrm{CF}$ and caregivers. Factors such as patient age, screening scores, clinical assessment, functional impairment and safety should be considered in developing a treatment plan. In addition, interventions should be adjusted to account for patient/caregiver preferences, medical status, psychiatric comorbidities, treatment history, resource availability, access to treatments and local practice patterns. See Recommendation 8 in table 1 .

The PHQ-9 and GAD-7 can be used both to screen these symptoms and to assess and monitor treatment response and adequacy of the treatment plan. If depression or anxiety symptoms continue to be elevated or functioning remains impaired 12 weeks later, additional or alternative interventions should be offered until symptoms return to within normal range.

Depending on the clinical context, the differential diagnosis in individuals with symptoms of depression and anxiety may include bipolar disorder, post-traumatic stress disorder, delirium, substance abuse or disease-related factors, such as fatigue, sleep disturbance, dyspnoea, pain $^{31}$ and vitamin D deficiency. ${ }^{32}$ Concomitant CF symptoms should be actively treated while depression and anxiety are being addressed. CF pulmonary exacerbations may worsen depression and anxiety through multiple biopsychosocial mechanisms, including the adverse impact of demoralisation, stress and inflammation. ${ }^{33}$

Considering the limited evidence for psychopharmacological treatment of depression and anxiety in children, psychological interventions tailored to individual and family needs are 


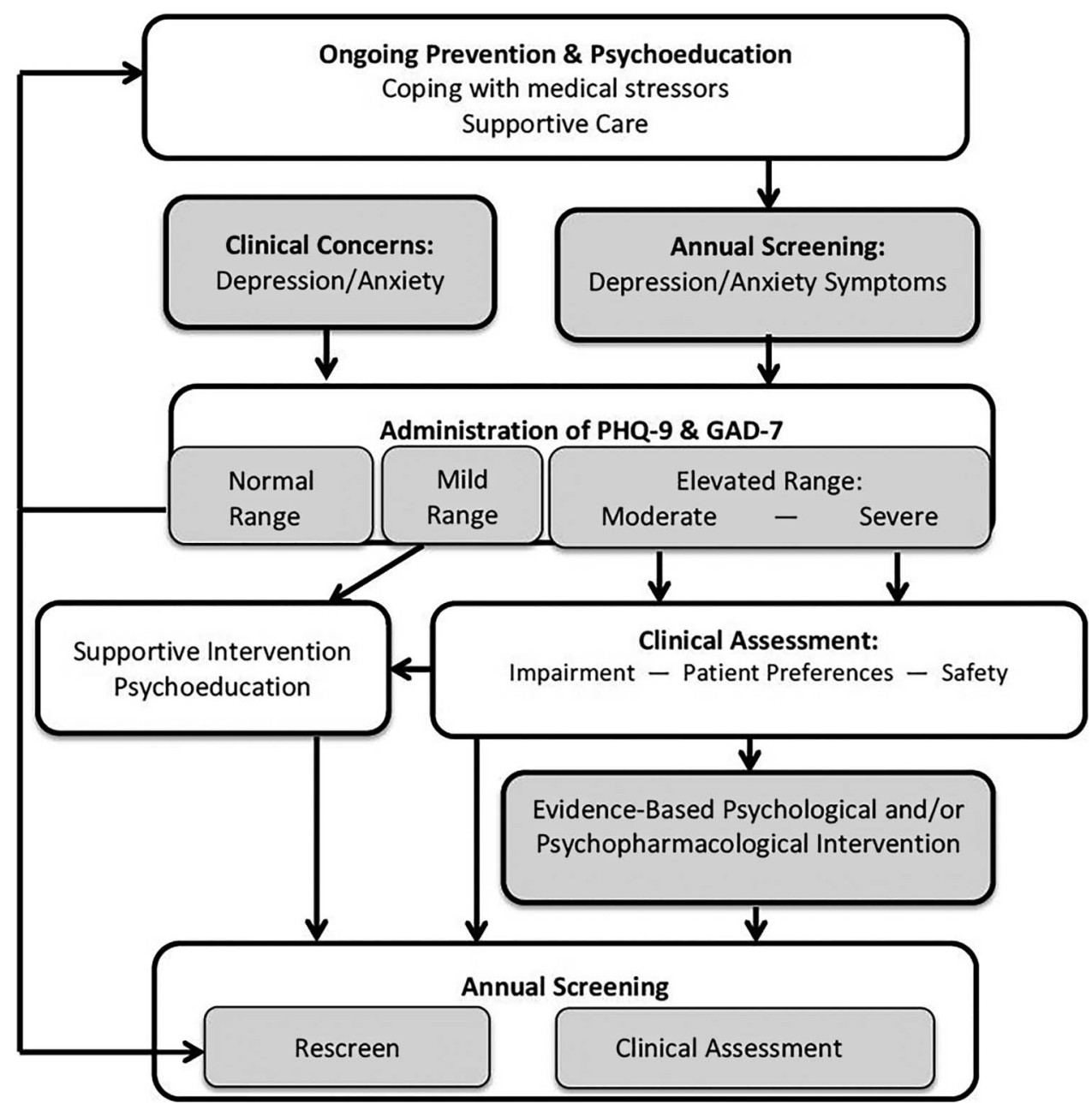

Figure 1 A flexible, stepped-care model for assessing and treating depression and anxiety.

recommended as the first-line approach in all children with CF ages 7-11 who require treatment. Specialised consultation should be obtained if psychological interventions are not sufficiently effective. See Recommendation 9 in table 1.

\section{Psychological interventions}

Adolescents and adults with CF (ages 12 and above) whose depression or anxiety is in the mild range should receive education, preventive or supportive psychological interventions and rescreening at the next $\mathrm{CF}$ visit. Evidence-based psychological interventions should be offered to all adolescents and adults with CF whose depression or anxiety is of at least moderate severity. An extensive body of literature supports the efficacy and effectiveness of cognitive behavioural therapy (CBT) for the treatment of both depression and anxiety, whereas some evidence indicates that interpersonal therapy (IPT) is an effective treatment for depression (see online supplementary appendix D tables 1-3). ${ }^{6}$ CBT is a psychotherapeutic approach that addresses dysfunctional emotions, behaviours and cognitions. It combines cognitive interventions (ie, challenge and replace negative thoughts with more functional cognitions) with the principles of behaviour modification (eg, training of skills and behaviours, using classical and operant learning principles). ${ }^{34}$ See Recommendations 10 and 11 in table 1.

Education and cognitive restructuring are included in CBT interventions for both depression and anxiety, whereas behavioural activation (eg, engaging in pleasant activities) is an additional core ingredient of most CBT manuals for the treatment of depression. Relaxation training and gradual exposure to triggers of anxiety are considered essential components of anxiety-specific CBT. IPT is a short-term treatment that encourages patients to regain control of mood and functioning. It is based on a treatment alliance in which the therapist empathically engages the patient, helps the patient feel understood and structures success experiences. ${ }^{35}$ Comparisons of CBT and IPT suggest there is broader empirical support, dissemination and training and worldwide adoption of CBT versus IPT. CBT has also demonstrated efficacy in treating comorbid depression and anxiety, which occur commonly among those with $\mathrm{CF}^{13}$

\section{Pharmacological interventions}

In individuals with CF, antidepressant medication should generally be prescribed in conjunction with psychological interventions, as part of a comprehensive treatment plan. For those with severe depression, evidence supports the combined use of antidepressants and psychological interventions as the most effective initial therapy. ${ }^{36}$ For adolescents and adults with CF who report moderate depression or moderate-to-severe anxiety, antidepressant medication should be considered when psychological interventions are not feasible or fully effective (see figures 2 and 3). See Recommendations 11, 12 and 13 in table 1.

When pharmacotherapy is needed, the selective serotonin reuptake inhibitors (SSRIs) citalopram, escitalopram, sertraline 


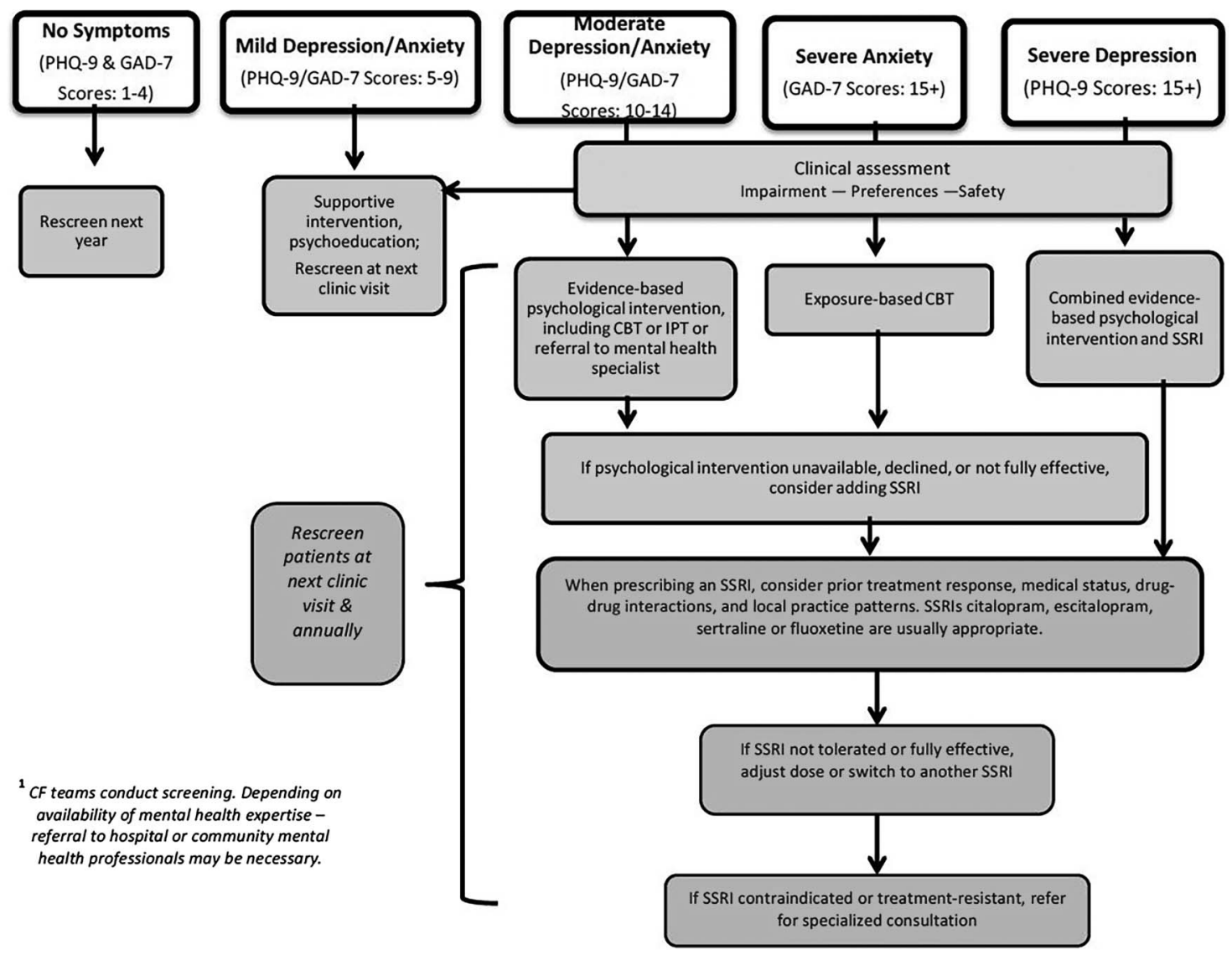

Figure 2 Screening and treatment of depression and anxiety: algorithm for individuals with cystic fibrosis (CF) (ages 12-adulthood). CBT, cognitive behavioural therapy; GAD-7, Generalised Anxiety Disorder 7-item Scale; IPT, interpersonal therapy; PHQ-9, Patient Health Questionnaire 9; SSRI, selective serotonin reuptake inhibitor.

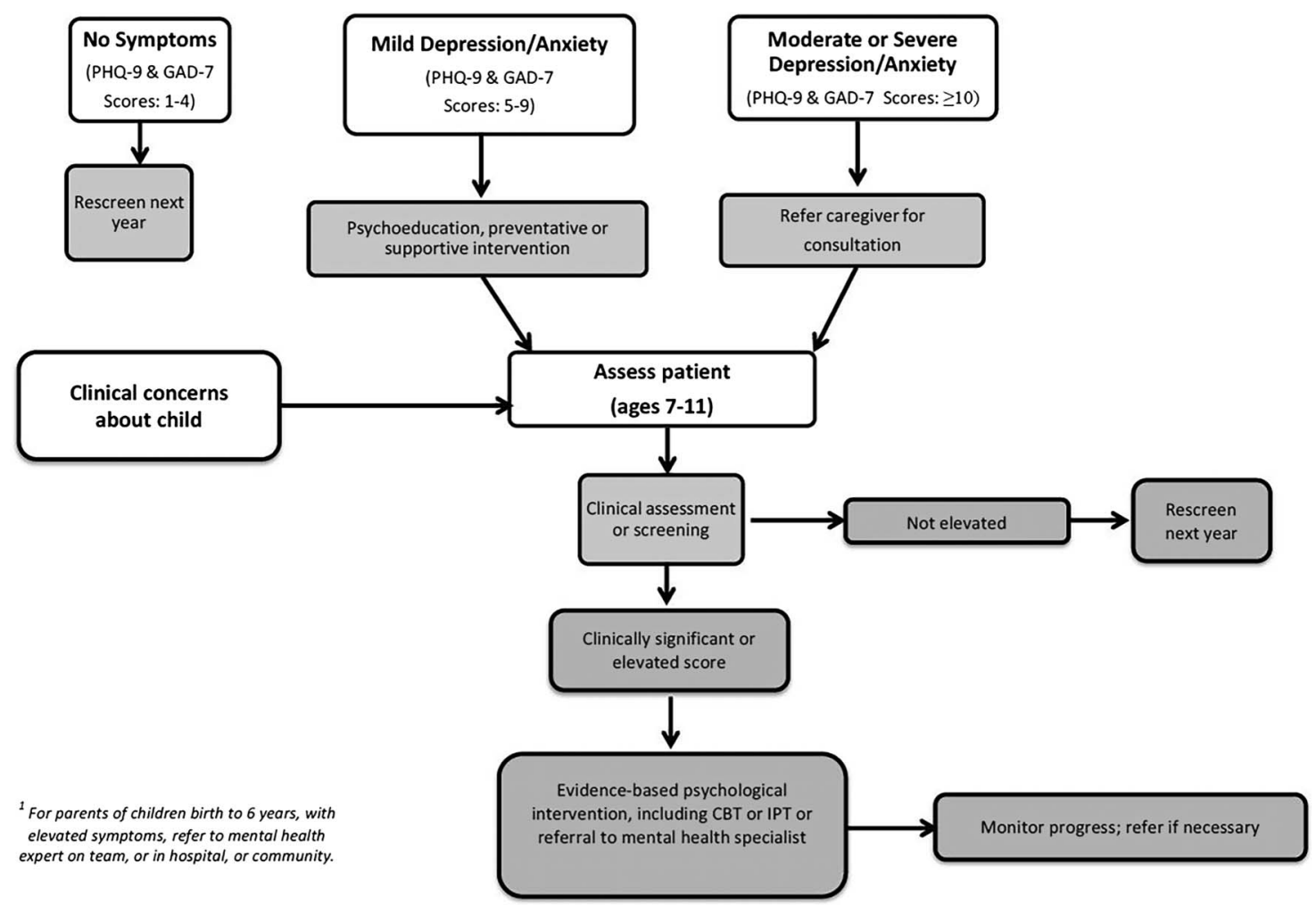

Figure 3 Screening and treatment of depression and anxiety: algorithm for parents/caregivers. CBT, cognitive behavioural therapy; GAD-7, Generalised Anxiety Disorder 7-item Scale; IPT, interpersonal therapy; PHQ-9, Patient Health Questionnaire 9. 
and fluoxetine are appropriate initial choices for most adolescents and adults with CF (see online supplementary appendix D and table 4). The ICMH sought to identify a short list of medications with strong evidence for their use in medical populations. SSRIs, although commonly referred to as antidepressants, are recommended by virtually all published guidelines as firstline medications for both depression and anxiety. Given the frequent co-occurrence of depression and anxiety in $\mathrm{CF}^{13}$ the effectiveness of SSRIs in treating either condition or both simultaneously is advantageous; this has not been demonstrated for many alternative antidepressants or antianxiety agents. Among the SSRIs and alternative agents, citalopram, escitalopram, sertraline and fluoxetine are more likely to be available inexpensively in many countries, covered by health plans, have regulatory approvals in a variety of age groups and minimise the potential for medication interactions and side effects. See Recommendation 14 in table 1.

Since the pharmacokinetics of medications may be altered in CF and may be variable across individuals, optimal dose adjustment of psychopharmacological agents requires close monitoring of therapeutic benefits, adverse effects and medical status. In CF, pulmonary, gastrointestinal/hepatic, renal and nutritional changes are particularly salient. Dose reduction may be required in individuals with renal or hepatic impairment, treatment-emergent adverse effects or drug-drug interactions. Dose increases may be required for those with impaired absorption or enhanced hepatic metabolism, partial response to treatment or drug-drug interactions. Therapeutic drug monitoring of blood levels, when available, may supplement clinical monitoring of psychotropic medication dosing. See Recommendation 14 in table 1.

To reduce the risk of drug-drug interactions, prescribing clinicians should be informed of all medications used daily, regularly cycled or used periodically for CF exacerbations. For example, when used with lumacaftor, the doses of citalopram, escitalopram and sertraline may need to be increased. Linezolid is not recommended for use with serotonergic antidepressants when alternatives are readily available. When both are clinically necessary, the lowest effective doses should be used, with informed consent and monitoring for serotonin syndrome. QTc prolongation, while not usually clinically significant, is more likely with citalopram than other SSRIs; electrocardiogram (EKG) and electrolyte monitoring can be considered when simultaneous use of multiple medications known to prolong the QTc is clinically necessary. ${ }^{37}$

\section{Anxiety in relation to medical procedures}

For moderate-to-severe episodic anxiety associated with medical procedures that have not responded to behavioural approaches, the benzodiazepine lorazepam may be considered for short-term use. Benzodiazepines require additional caution and monitoring for those with a history of substance abuse, depression or an elevated risk for respiratory depression, and local practice patterns differ regarding their use to treat anxiety. Benzodiazepines are preferable to SSRIs primarily when rapid onset of action is needed or when serotonergic agents are contraindicated. Lorazepam is available in both oral and intravenous forms, and its duration of action is short enough to avoid a prolonged period of sedation following a procedure. It is renally excreted with no hepatic metabolism, which reduces medication interactions and may be useful in the context of CF liver disease. However, dose reduction may be necessary in patients who develop renal insufficiency. See Recommendation 15 in table 1.
Medications used for treatment-resistant depression or anxiety, which may carry increased risks of drug-drug interactions and adverse effects in individuals with $\mathrm{CF}$, are outside the scope of this guideline. When necessary, they should be prescribed and monitored by a psychiatric specialist in close collaboration with the CF team. Specialised consultation should also be obtained when the psychiatric diagnosis is uncertain, the complexity of the case exceeds the CF team's level of training and experience or when an urgent safety risk is identified.

\section{RECOMMENDATIONS FOR IMPLEMENTATION AND FUTURE RESEARCH}

As screening for depression and anxiety is implemented in CF centres internationally, the process of dissemination, implementation and resource provision should be closely monitored to address barriers and concerns. As many international healthcare providers do not have a colleague trained in mental health, ${ }^{22}$ implementation will require providing $\mathrm{CF}$ healthcare professionals with (1) training, easy access to resources and a 'toolkit' to facilitate implementation of annual mental health screening (see online supplementary appendix D); (2) training in the provision of preventive and supportive interventions and (3) development of referral networks within the hospital and community for subsequent psychological and/or pharmacological management of clinically diagnosed depression and anxiety. Many initiatives directly tied to these recommendations are in process. For example, the CFF in the USA recently convened a Task Force on Mental Health, which outlined both the challenges and the importance of implementing these new guidelines, is sponsoring short courses and conference sessions on this topic at the North American CF Conference and is launching a 'request for applications' to provide resources to $\mathrm{CF}$ centres that implement screening and treatment. Addressing mental health issues systematically is likely to improve health outcomes, quality of life and reduce healthcare utilisation. ${ }^{1038}$

Despite evidence in the TIDES study ${ }^{13}$ that a new diagnosis of CF-related diabetes, and events such as haemoptysis and pneumothorax, may precede elevations in symptoms of depression and anxiety, little is also known about the 'triggers' of psychological symptoms, or how mood affects health outcomes in this complex disease. The development of preventative strategies will be enhanced by research which identifies psychosocial and clinical risk factors, such as maladaptive coping, social isolation, CF clinical complications, markers of inflammation or vitamin deficiencies that predict the onset of depression or anxiety in individuals with CF. Large-scale studies are also required to examine how psychological symptoms affect disease management (eg, adherence) and health outcomes. In terms of cutting edge questions, new evidence in other chronic conditions (eg, cardiovascular disease) indicates that depression, independent of disease state, can increase inflammation and lead directly to a worsening of disease. ${ }^{39}$ New studies in CF are actively testing this hypothesis, which if supported, adds urgency to the initiation of both screening and treatment.

Although psychotherapy and medication treatments for depression and anxiety are already used in $\mathrm{CF}^{13}{ }^{14}$ these interventions require further systematic research to inform future recommendations. A recent Cochrane Review of psychological treatments for individuals with $\mathrm{CF}$ found no controlled studies evaluating interventions that address depression and anxiety in either individuals with CF or parent caregivers. ${ }^{30}$ Randomised, comparative effectiveness studies of psychotherapeutic and psychopharmacological interventions for depression and anxiety in CF are recommended. Additional studies are needed to 
understand not only the efficacy of cognitive behavioural interventions, but also the pharmacokinetics, the frequency of gastrointestinal or pulmonary side effects or beneficial weight gain. To understand the full potential of psychological and pharmacological treatments to improve care, beyond the reduction of symptoms of depression and anxiety, studies evaluating changes in adherence to CF treatments and/or medical outcomes, as well as the indirect effects of mental health treatment on parental caregivers, should be investigated. Multicentre studies are recommended to increase sample sizes and enhance statistical power in trials of a rare condition, such as $\mathrm{CF}$.

Finally, the inclusion of screening data, as well as intervention efforts, in our national registries will greatly facilitate answers to such pressing questions, including (1) evaluation of the prevalence of psychological symptoms by age, gender and disease severity, (2) identification of the predictors of elevated symptoms, (3) outcomes of both the screening and intervention processes, (4) consequences of psychological symptoms on adherence and health outcomes and (5) impact of psychological distress on healthcare utilisation and cost. Novel forms of service delivery, such as integrated care models or internet-based education and treatment, are also worthy of investigation. ${ }^{40}{ }^{41}$

\section{CONCLUSION}

Robust evidence indicates that adolescents, adults with CF and parent caregivers report elevations in depression and anxiety that should be identified and treated. Data also suggest that these symptoms affect both disease management and health outcomes. Systematic screening of these symptoms, with appropriate intervention, offers the opportunity to significantly improve the quality of life and health of individuals with CF and their parent caregivers.

\author{
Author affiliations \\ ${ }^{1}$ University of Miami, Coral Gables, Florida, USA \\ ${ }^{2}$ School of Psychology, University of Central Lancashire, Preston, UK \\ ${ }^{3}$ Department of Child and Adolescent Psychiatry, Massachusetts General Hospital, \\ Boston, Massachusetts, USA \\ ${ }^{4}$ Department of Child and Adolescent Psychiatry/Psychotherapy, University Hospital \\ Ulm, Ulm, Germany \\ ${ }^{5}$ Division of Child and Adolescent Psychiatry, State University of New York, Buffalo, \\ New York, USA \\ ${ }^{6}$ The Dartmouth Institute for Health Policy and Clinical Practice, Geisel School of \\ Medicine at Dartmouth, Lebanon, New Hampshire, USA \\ ${ }^{7}$ Cystic Fibrosis Foundation, Bethesda, Maryland, USA \\ ${ }^{8}$ Queen's University, Belfast, UK
}

Collaborators IC MH: Janice Abbott, BA PhD, School of Psychology University of Central Lancashire, Preston PR1 2HE, UK. Michael Boyle, MD, Johns Hopkins University School of Medicine. Amanda Crossan, DPhil, DClinPsych, Northern Ireland Regional Adult Cystic Fibrosis Centre. Stuart Elborn, MD, Queen's University Belfast, 97 Lisburn Road, Belfast BT9 7BL, UK. Silvia Gartner, MD, Hospital Universitari Vall d'Hebron, Barcelona, Spain. Anna Georgiopoulos, MD, Mass General Hospital, and Harvard School of Medicine, Boston, MA, USA. Manfred Gerlach, PhD, University Hospital Würzburg, Würzburg, Germany. Lutz Goldbeck, PhD, University Hospital Ulm, Ulm, Germany. Sarah Hempstead, MS, Jennifer Rounds, RN, BSN, Kathryn A Sabadosa, MPH, The Dartmouth Institute for Health Policy and Clinical Practice, Geisel School of Medicine at Dartmouth, Lebanon, NH, USA. Martin Hug, PhD, University Medical Center Freiburg, Freiburg, Germany. Christopher Kvam, JD, Michelle Macias, MD, Medical University of South Carolina, Charleston, SC, USA. Bruce Marshall, MD, CFF, Bethesda, MD, USA. Amy Mueller, MSW LCSW, Hartford Hospital, Hartford, CT, USA. Jacquelien Noordhoek, MA, MSC, Dutch CF Foundation, Baarn, The Netherlands. Alexandra Quittner, PhD, University of Miami, Coral Gables, FL, USA. Paul Ramchandi, BM, DPhil, Imperial College, London. Kim Reno, MSW, LISW, Pediatric Pulmonary and Cystic Fibrosis Care Center of Northwest Ohio, Toledo, OH, USA. Kristin Riekert, PhD, Johns Hopkins School of Medicine, Baltimore, MD, USA. Beth Smith, MD, State University of New York at Buffalo, Buffalo, NY, USA. Ahmet Uluer, DO MS, Boston Children's and Brigham and Women's Hospital, Harvard Medical School, Boston, MA, USA. Jacquelyn Zirbes, DNP, RN, CNP, CCRC, Stanford University, Stanford, CA, USA. IC MH: Michael Boyle, Amanda Crossan,
Silvia Gartner, Manfred Gerlach, Martin Hug, Christopher Kvam, Michelle Macias, Amy Mueller, Jacquelien Noordhoek, Paul Ramchandani, Kim Reno, Kristin Riekert, Jennifer Rounds, Ahmet Uluer and Jacquelyn Zirbes.

Contributors The CFF and ECFS sponsored the work of the ICMH for over 2 years. ALQ and JSE co-chaired the ICMH. ALQ reviewed and critiqued the epidemiological studies of depression and anxiety in individuals with chronic conditions and their parents and caregivers. BS reviewed and critiqued the literature on screening measures that are free and available in all major languages. LG and AMG reviewed and critiqued the literature on evidence-based interventions to treat depression and anxiety. JA conducted a survey of best practices for healthcare providers in mental health in the USA and Europe. JA and BM reviewed and critiqued the literature on dissemination of mental health interventions. SEH and KAS provided assistance on PICO guidelines and literature searches. The members of the ICMH participated in committees to gather relevant information and literature to analyse and critique. All of the authors voted on the consensus recommendations and statements, contributed to the writing and review of the manuscript and preparation and editing of online supplementary appendices.

Funding The authors received funding from the CFF and the ECFS.

Competing interests The authors received support from the CFF and the ECFS during the conduct of this work. ALQ reports investigator-initiated grants from Novartis and consulting income from Vertex Pharmaceuticals and Abbvie Pharmaceuticals, outside the submitted work. JA, BS, SEH, BM, KAS have nothing to disclose. AMG reports personal fees from Lippincott, Williams \& Wilkins, outside the submitted work. LG reports consultancy fees paid to his institution from Vertex Pharmaceuticals, outside the submitted work. SE reports clinical trial and consultancy fees paid to his institution from Vertex Pharmaceuticals, Novartis, Allergan and Gilead, outside the submitted work.

Provenance and peer review Not commissioned; externally peer reviewed.

Open Access This is an Open Access article distributed in accordance with the Creative Commons Attribution Non Commercial (CC BY-NC 4.0) license, which permits others to distribute, remix, adapt, build upon this work non-commercially, and license their derivative works on different terms, provided the original work is properly cited and the use is non-commercial. See: http://creativecommons.org/ licenses/by-nc/4.0/

\section{REFERENCES}

1 Pinquart M, Shen Y. Depressive symptoms in children and adolescents with chronic physical illness: an updated meta-analysis. J Pediatr Psychol 2011;36:375-84.

2 Moussavi S, Chatterji S, Verdes E, et al. Depression, chronic diseases, and decrements in health: results from the World Health Surveys. Lancet 2007;370:851-8.

3 Mall MA, Elborn JS. ERS monograph: cystic fibrosis. Sheffield, UK: European Respiratory Society, 2014.

4 Sawicki GS, Sellers DE, Robinson WM. High treatment burden in adults with cystic fibrosis: challenges to disease self-management. J Cyst Fibros 2009;8:91-6.

5 Joffres M, Jaramillo A, Dickinson J, et al., Canadian Task Force on Preventative Health. Recommendations on screening for depression in adults. CMAJ 2013; 185:775-82.

6 National Collaborating Center for Mental Health. The NICE guideline on the management and treatment of depression in adults (updated edition). Leicester, UK: British Psychological Society, 2010.

7 Cystic Fibrosis Foundation Patient Registry. 2012 Annual Data Report, 2012.

8 American Psychiatric Association, ed. Diagnostic and Statistical Manual of Mental Disorders (DSM-5). 5th edn. American Psychiatric Publishing, Incorporated, 2013.

9 Uman LS, Birnie KA, Noel M, et al. Psychological interventions for needle-related procedural pain and distress in children and adolescents. Cochrane Database Syst Rev 2013;10:CD005179.

10 Smith BA, Modi AC, Quittner AL, et al. Depressive symptoms in children with cystic fibrosis and parents and its effects on adherence to airway clearance. Pediatr Pulmonol 2010;45:756-63.

11 Latchford G, Duff AJ. Screening for depression in a single CF centre. J Cyst Fibros 2013;12:794-6.

12 Havermans T, Colpaert K, Dupont LJ. Quality of life in patients with cystic fibrosis: association with anxiety and depression. J Cyst Fibros 2008;7:581-4.

13 Quittner AL, Goldbeck L, Abbott J, et al. Prevalence of depression and anxiety in patients with cystic fibrosis and parent caregivers: results of the International Depression Epidemiological Study across nine countries. Thorax 2014;69:1090-7.

14 Ploessl C, Pettit RS, Donaldson J. Prevalence of depression and antidepressant therapy use in a pediatric cystic fibrosis population. Ann Pharmacother 2014;48:488-93.

15 Snell C, Fernandes S, Bujoreanu IS, et al. Depression, illness severity, and healthcare utilization in cystic fibrosis. Pediatr Pulmonol 2014;49:1177-81.

16 Hilliard ME, Eakin ME, Borrelli B, et al. Medication beliefs mediate between depressive symptoms and medication adherence in cystic fibrosis. Health Psycho/2015;34:496-504. 
17 Riekert KA, Bartlett SJ, Boyle MP, et al. The association between depression, lung function, and health-related quality of life among adults with cystic fibrosis. Chest 2007;132:231-7.

18 Robinson KA, Saldanha IJ, McKoy NA. Development of a framework to identify research gaps from systematic reviews. J Clin Epidemiol 2011;64:1325-30.

19 Borowitz D, Robinson KA, Rosenfeld M, et al. Cystic Fibrosis Foundation evidence-based guidelines for management of infants with cystic fibrosis. J Pediatr 2009;155:S73-93.

20 Smyth AR, Bell SC, Bojcin S, et al. European Cystic Fibrosis Society standards of care: best practice guidelines. J Cyst Fibros 2014;13(Suppl 1):S23-42

21 O'Connell ME, Boat T, Warner KE, eds. Preventing Mental, Emotional, and Behavioral Disorders Among Young People: Progress and Possibilities. Washington DC: National Academies Press, 2009.

22 Abbott J, Elborn S, Georgiopoulos A, et al. Cystic Fibrosis Foundation and European Cystic Fibrosis Society survey of cystic fibrosis mental health care delivery. J Cyst Fibros 2015;14:533-9.

23 U.S. Preventive Services Task Force. Screening for depression in adults: U.S. Preventive Services Task Force recommendation statement. Ann Intern Med 2009;151:784-92.

24 U.S. Preventive Services Task Force. Screening and treatment for major depressive disorder in children and adolescents: U.S. Preventive Services Task Force recommendation statement. Pediatrics 2009;123:1223-8.

25 Geoffrey RS, Cynthia B, Graham AB III, et al. 2014 recommendations for pediatric preventive health care. Pediatrics 2014;133:568-70.

26 National Collaborating Center for Mental Health. Common Mental Health Disorders: The NICE Guideline on identification and pathways to care. Leicester, UK: The British Psychological Society \& The Royal College of Psychiatrists, 2011.

27 National Collaborating Center for Mental Health. Depression in adults with a chronic health problem: treatment and management. Leicester, UK: National Institute for Health and Clinical Excellence, 2009.

28 Andersen $B$, DeRubeis $R$, Berman B, et al. Screening, assessment, and care of anxiety and depressive symptoms in adults with cancer: an American Society of Clinical Oncology guideline adaptation. J Clin Oncol 2014; 32:1605-19.
29 Posner K, Brown GK, Stanley B, et al. The Columbia-Suicide Severity Rating Scale; initial validity and internal consistency findings from three multisite studies with adolescents and adults. Am J Psychiatry 2011;168:1266-77.

30 Goldbeck L, Fidika A, Herle M, et al. Cochrane corner: psychological interventions for individuals with cystic fibrosis and their families. Thorax 2015;70:1089-91.

31 Blackwell LS, Quittner AL. Daily pain in adolescents with CF: effects on adherence, psychological symptoms, and health-related quality of life. Pediatr Pulmonol 2015;50:244-51

32 Smith BA, Cogswell A, Garcia G. Vitamin D and depressive symptoms in children with cystic fibrosis. Psychosomatics 2014;55:76-81.

33 Felger JC, Lotrich FE. Inflammatory cytokines in depression: neurobiological mechanisms and therapeutic implications. Neuroscience 2013;246:199-229.

34 Rachman S. The evolution of cognitive behaviour therapy. In: Clark DF, Fairburn CG, Gelder MG, eds. Science and practice of cognitive behaviour therapy. Oxford, UK: Oxford University Press, 1997:1-26.

35 Cornes CL, Frank E. Interpersonal psychotherapy for depression. Clin Psychol 1994:47:9-10.

36 March JS, Vitiello B. Clinical messages from the treatment for adolescents with depression study (TADS). Am J Psychiatry 2009;166:1118-23.

37 Beach SR, Celano CM, Noseworthy PA, et al. QTc prolongation, torsades de pointes, and psychotropic medications. Psychosomatics 2013;54:1-13.

38 Burker EJ, Sedway J, \& Carone S. Psychological and educational factors: better predictors of work status than FEV1 in adults with cystic fibrosis. Pediatr Pulmonol 2004;38:413-18

39 Berk M, Williams LJ, Jacka FN, et al. So depression is an inflammatory disease, but where does the inflammation come from? BMC Med 2013;11:200.

40 Fidika A, Herle M, Lehmann C, et al. A web-based psychological support program for caregivers of children with cystic fibrosis: a pilot study. Health Qual Life Outcomes 2013;11:1-9.

41 Huffman JC, Niazi SK, Rundell JR, et al. Essential articles on collaborative care models for the treatment of psychiatric disorders in medical settings: a publication by the Academy of Psychosomatic Medicine Research and Evidence-Based Practice Committee. Psychosomatics 2014;55:109-22. 